\title{
Dynamic Time Course of Typical Childhood Absence Seizures: EEG, Behavior, and Functional Magnetic Resonance Imaging
}

\author{
Xiaoxiao Bai, ${ }^{1}$ Matthew Vestal, ${ }^{1}$ Rachel Berman, ${ }^{1}$ Michiro Negishi, ${ }^{2}$ Marisa Spann, ${ }^{5}$ Clemente Vega, ${ }^{5}$ Matthew Desalvo, ${ }^{1}$ \\ Edward J. Novotny, ${ }^{1,3,5}$ Robert T. Constable, ${ }^{2}$ and Hal Blumenfeld ${ }^{1,4,5}$ \\ Departments of ${ }^{1}$ Neurology, ${ }^{2}$ Diagnostic Radiology, ${ }^{3}$ Pediatrics, ${ }^{4}$ Neurobiology, and ${ }^{5}$ Neurosurgery, Yale University School of Medicine, New Haven, \\ Connecticut 06520
}

\begin{abstract}
Absence seizures are 5-10 s episodes of impaired consciousness accompanied by 3-4 Hz generalized spike-and-wave discharge on electroencephalography (EEG). The time course of functional magnetic resonance imaging (fMRI) changes in absence seizures in relation to EEG and behavior is not known. We acquired simultaneous EEG-fMRI in 88 typical childhood absence seizures from nine pediatric patients. We investigated behavior concurrently using a continuous performance task or simpler repetitive tapping task. EEG timefrequency analysis revealed abrupt onset and end of $3-4 \mathrm{~Hz}$ spike-wave discharges with a mean duration of $6.6 \mathrm{~s}$. Behavioral analysis also showed rapid onset and end of deficits associated with electrographic seizure start and end. In contrast, we observed small early fMRI increases in the orbital/medial frontal and medial/lateral parietal cortex $>5 \mathrm{~s}$ before seizure onset, followed by profound fMRI decreases continuing $>20 \mathrm{~s}$ after seizure end. This time course differed markedly from the hemodynamic response function (HRF) model used in conventional fMRI analysis, consisting of large increases beginning after electrical event onset, followed by small fMRI decreases. Other regions, such as the lateral frontal cortex, showed more balanced fMRI increases followed by approximately equal decreases. The thalamus showed delayed increases after seizure onset followed by small decreases, most closely resembling the HRF model. These findings reveal a complex and long-lasting sequence of fMRI changes in absence seizures, which are not detectable by conventional HRF modeling in many regions. These results may be important mechanistically for seizure initiation and termination and may also contribute to changes in EEG and behavior.
\end{abstract}

\section{Introduction}

Absence seizures are brief 5-10 s episodes of impaired consciousness and 3-4 Hz generalized spike-wave discharges, which begin and end abruptly on electroencephalography (EEG) (Blumenfeld, 2005b; Andermann, 2006). Despite the fact that electrical events and behavioral changes in absence seizures have been investigated thoroughly (Davidoff and Johnson, 1964; Mirsky and Van Buren, 1965; Sadleir et al., 2006, 2009), shortcomings of scalp EEG in both the spatial resolution and detection of subcortical regions have limited the understanding of fundamental mechanisms of altered brain function in absence seizures.

In the past decade, functional magnetic resonance imaging (fMRI) with simultaneous EEG recording has been increasingly used in generalized epilepsy studies (Archer et al., 2003; Salek-

Received 0ct. 11, 2009; revised Jan. 26, 2010; accepted Jan. 29, 2010.

This work was supported by National Institutes of Health (R01 NS055829, CTSA UL1 RR0249139, and MSTP TG 5T32GM07205) and by the Betsy and Jonathan Blattmachr family. We thank N. Danielson and B. Killory for comments on the manuscript. We also thank the following clinicians who referred patients for the study: A. Bhargava, B. Bourgeois, W. Brown, G. Castaneda, R. L. Cerciello, R. Cheng, F. DiMario, R. B. Duckrow, M. Engel, J. Gaitanis, J. Gibbons, L. Kan, S. R. Levy, D. Mandelbaum, G. Miller, S. Moshe, S. Nallainathan, P. Overby, S. Rothman, R. Smith, Y. Sogawa, F. Testa, S. Wolf, and R. Young.

Correspondence should be addressed to Dr. Hal Blumenfeld, Yale Department of Neurology, 333 Cedar Street, New Haven, CT 06520. E-mail: hal.blumenfeld@yale.edu.

DOI:10.1523/JNEUROSCI.5101-09.2010

Copyright $\odot 2010$ the authors $\quad 0270-6474 / 10 / 305884-10 \$ 15.00 / 0$
Haddadi et al., 2003; Aghakhani et al., 2004; Gotman et al., 2005; Labate et al., 2005; Hamandi et al., 2006; Laufs et al., 2006; Hawco et al., 2007; Moeller et al., 2008a,b; Vaudano et al., 2009), revealing the cortical and subcortical areas with blood oxygenation level-dependent (BOLD) changes. Several groups have used this EEG-fMRI approach to investigate generalized spike-wave discharges, and their findings have demonstrated consistent bilateral fMRI increases in thalamus and decreases in medial and lateral parietal cortex; however, changes in other cortical and subcortical regions have been more variable.

Recent fMRI studies in normal subjects have provided important evidence that the actual hemodynamic response may vary from one brain area to another (Miezin et al., 2000; Neumann et al., 2003; Handwerker et al., 2004; Meltzer et al., 2008), which may lead to highly variable findings in model-driven fMRI analyses. An EEG/MEG study in epileptic patients (Amor et al., 2009) reported local and long-range synchronization a few hundred milliseconds before spike-wave discharges, while recent fMRI studies have found changes up to several seconds before electrical epileptiform activity (Hawco et al., 2007; Moeller et al., 2008a). These findings suggest that the longer-lasting BOLD response dynamics in absence seizures may be variable for different regions and could occur even before spike-wave discharges on EEG. Furthermore, a recent modeling study supported the possible role of specific cortical regions such as the medial parietal cortex (pre- 
cuneus) in initiating fMRI changes with spike-wave discharges (Vaudano et al., 2009).

Although models can provide valuable insights, we were interested in directly examining the fMRI time course in absence seizures using a data-driven approach. To accomplish this, we studied a relatively homogenous group of children with typical childhood absence epilepsy, and first analyzed the time course of EEG and behavioral changes before, during, and after seizures. Next, we analyzed simultaneously recorded fMRI signals using conventional HRF modeling. Finally, we performed a direct analysis of mean fMRI time courses in a relatively large number of seizures both in the whole brain, and in specific regions showing maximal changes during the time period from $-20 \mathrm{~s}$ to $+40 \mathrm{~s}$ relative to seizure onset. This approach allowed us to characterize a complex sequence of early and late fMRI changes, not detectable by conventional analysis, which may shed new light on absence seizure pathogenesis.

\section{Materials and Methods}

Patients. From January 2004 to February 2009, 42 pediatric patients with typical childhood absence epilepsy were referred by their pediatric neurologists. All patients had informed consent signed by their legal guardians, and all human study procedures were approved by the institutional review boards at Yale University School of Medicine and the Yale Magnetic Resonance Imaging Center. Of these 42 patients, 9 had absence seizures during EEG-fMRI and were used for analysis. Patients fulfilled the following criteria: (1) clinical diagnosis of childhood absence epilepsy based on International League Against Epilepsy criteria (Commission on Classification and Terminology of the International League Against Epilepsy, 1989); (2) EEG with typical 3-4 Hz bilateral spike-wave discharges and normal background activity; (3) age range of 6-18 years; (4) no additional seizure types, such as myoclonic, tonic-clonic, or partial seizures; (5) no known structural brain abnormality; and (6) no other neurological disorders.

Experimental procedure. Before actual EEG-fMRI scanning, patients were familiarized with the MRI environment in an early separate visit. All pediatric patients were trained for the behavioral tests and underwent at least one practice session in a mock scanner after training. To increase the likelihood of capturing absence seizures during the EEG-fMRI scans, patients stopped taking medication for up to $48 \mathrm{~h}$ before scanning (Buchanan et al., 1969; Hvidberg and Dam, 1976; Barceloux, 1998; Chen et al., 1999). Though some activating procedures, such as hyperventilation, sleep deprivation, or photic stimulation, may increase the chances of absence seizures, they were not used in this study. Hyperventilation causes cerebral vasoconstriction, which would complicate interpretation of fMRI results, photic stimulation-induced seizures may differ from spontaneous absence seizures, and sleep deprivation may impair behavioral performance on the vigilance testing.

Behavioral tasks and analysis. Two behavioral tasks and a visual fixation task were performed during the EEG-fMRI acquisitions for each patient. The behavioral tasks we used have been used previously (Yeager and Guerrant, 1957; Jus and Jus, 1960; Mirsky and Van Buren, 1965) to examine the impairment of consciousness in patients with typical absence epilepsy, providing valuable information about deficits during and between seizures. To determine fMRI changes in the same patients during spike-wave discharges but without any active task, a visual fixation run was also acquired in most patients. All tasks were generated using E-Prime 1.1 (Psychology Software Tools). One fMRI run for each task consisted of 20 blocks (each block $=32 \mathrm{~s}$ ), and lasted for a total of $10 \mathrm{~min}$ and $40 \mathrm{~s}$.

Tasks were as follows: (1) The continuous performance task (CPT) was composed of 10 blocks of visual stimuli alternating with 10 blocks of fixation (the first block was fixation). During stimuli blocks, the following 16 letters were displayed in random sequence: A B C D E F H I L M N O T X Y Z. Twenty-five percent of all letters shown were the target X, so that the $\mathrm{X}$ appeared on average every $4 \mathrm{~s}$. The letters were displayed for $250 \mathrm{~ms}$, and the interval between consecutive letter onsets was $1000 \mathrm{~ms}$, allowing 750 ms between letters. Patients responded to the target letter X by using the right hand to push a button. During fixation blocks, patients viewed a + sign presented on the screen. (2) The repetitive tapping task (RTT) was the same as the CPT except patients were instructed to push the button for every displayed letter (once per second), and no letter X appeared in the sequence. (3) The visual fixation task (VFT) consisted of 20 uniform fixation blocks. Patients viewed the fixation sign + during the entire 10 min $40 \mathrm{~s}$ run.

For all tasks, the letters and + sign appeared on a projection screen, viewed by a mirror mounted on the patient head coil. Both the stimuli and button push response times for displayed letters were recorded using the E-Prime program. The intervals for correct responses were 120-1000 ms after stimulus presentation for CPT and $0-1000 \mathrm{~ms}$ after stimulus presentation for RTT. Performance on the CPT and RTT was analyzed by calculating the correct response percentage rate, $(\mathrm{A} 1 / \mathrm{A} 0) \times 100$, where $\mathrm{A} 0$ was the number of target letters and $\mathrm{A} 1$ was the number of correct responses for target letters. For a given patient, the correct response percentage rate for the RTT or CPT was calculated in two second bins across runs, and the mean CPT/RTT performance rates at each time were then calculated by averaging across subjects.

EEG-fMRI data acquisition. Continuous EEG-fMRI data were acquired from each patient. Spike-wave discharges of typical absence seizures were found on EEG data from nine patients. EEG data were acquired with two slightly different systems. The EEG data in seven of these nine patients were collected by an EEG cap with silver/silverchloride electrodes (modified from Quik-Cap 21 channel (international 10-20 system), Neuroscan), carbon fiber cables (in-house), a $125 \mathrm{~Hz}$ analog low-pass Butterworth filter (in-house), and an EEG recorder (NuAmps, Neuroscan). EEG signals were recorded at a $500 \mathrm{~Hz}$ sampling rate with 22 bit data resolution and referred to a linked ear reference. The EEG data in the two other patients were recorded using the same system except that 32 carbon wire EEG electrodes (in-house) and a preamplifier (in-house) were used (Negishi et al., 2008), and the signals were digitized at $1000 \mathrm{~Hz}$ with a newer EEG recorder with 24 bit data resolution (SynAmps2, Neuroscan).

All patients were scanned using a 3 Tesla Magnetom Trio scanner (Siemens Medical Systems). During scanning, foam padding was used to help secure the EEG leads, reduce motion artifacts, and improve patient comfort. An AC-PC-aligned axial $\mathrm{T}_{1}$-weighted anatomical scan was acquired using a spin-echo (SE) acquisition (repetition time $=300 \mathrm{~ms}$, echo time $=2.47 \mathrm{~ms}$, matrix size $=256 \times 256,25$ slices per image, slice thickness $=6 \mathrm{~mm}$, field of view $=22 \mathrm{~cm}$ ). Functional MRI images were recorded with an echo-planar imaging (EPI) sequence with the following parameters: repetition time $=1550 \mathrm{~ms}$, echo time $=30 \mathrm{~ms}$, flip angle $=$ 80 , matrix size $=64 \times 64$, and other parameters were the same as for the $\mathrm{T}_{1}$-weighted anatomical images, acquired in the same imaging planes. At the beginning of each fMRI scan, a TTL pulse from the MRI scanner was used to initiate the visual stimulus presentation. To further ensure correct synchronization, the behavioral stimulus presentations and button push responses were recorded along with the EEG data in separate channels, as well as by the stimulus presentation software. Each fMRI run lasted $10 \mathrm{~min}$ and $40 \mathrm{~s}$. Up to six (typically three or four) fMRI runs were obtained per recording session as tolerated by the patients.

EEG analysis. EEG data were filtered offline to remove the artifact generated by MRI scans, allowing the visualization of the entire EEG trace. The MR artifact in seven of nine patients was filtered using the SCAN (Neuroscan) software and in-house temporal principal component analysis (PCA) software (Negishi et al., 2004). The MR artifact in the remaining two patients was subtracted using adaptive noise cancellation software (Negishi et al., 2008). After artifact removal, the EEG data were low-pass filtered at $25 \mathrm{~Hz}$ and visually inspected.

Before all other EEG, behavioral, and fMRI analysis, EEG recordings were reviewed by an experienced reader (H.B.) to identify all typical 3-4 $\mathrm{Hz}$ spike-wave discharges, and to determine seizure onset and end times with $0.1 \mathrm{~s}$ time resolution. These times were used for all subsequent EEG, behavioral, and fMRI analyses.

A time-frequency analysis (Oppenheim and Schafer, 1989) was executed to investigate the time-frequency dynamics before, during, and after spike-wave discharge bursts. Analysis was performed on EEG data 
from $-20 s$ to $+40 s$ relative to each seizure onset. Time-frequency dynamics were calculated by using the short-time Fourier transform method in MATLAB 7.1 (MathWorks). The short-time Fourier transform includes a sliding time window (window width $=5.12 \mathrm{~s}$, shifted at $20 \mathrm{~ms}$ intervals), providing the frequency composition of short sequential data segments over time. The frequency band $0-25 \mathrm{~Hz}$ was investigated in each channel. Since low-frequency $(<1 \mathrm{~Hz})$ EEG signal was observed at all times independent of seizures, it was removed using a high-pass filter $(>1 \mathrm{~Hz})$. The ictal, preictal (times before seizure onset), and postictal (times after seizure end) time periods of EEG recordings were temporally aligned across seizures, and the ictal period of each seizure was scaled to the mean seizure duration $(6.6 \mathrm{~s})$. It should be noted that the preictal and postictal time data were not scaled; they were simply aligned in time across seizures by lining up the seizure onset times for preictal data, and by lining up the seizure end times for postictal data. The power spectrum for each seizure was normalized to the range [0 1$]$, so that the maximum power for each seizure was defined as "1." Finally, the aligned time-frequency dynamics in each channel were averaged across seizures for each patient, and the mean was calculated across patients.

fMRI analysis. The SPM2 software package (http://www.fil.ion.ucl.ac. $\mathrm{uk} / \mathrm{spm} /$ ) was used for all fMRI image preprocessing on a MATLAB 7.1 platform (MathWorks). The initial 10 images (15.5 s) were discarded in each run. The remaining 406 images in each run were spatially realigned to the first image of each functional series and spatially normalized to the SPM EPI template in MNI space. Images were then spatially smoothed using an isotropic Gaussian kernel (10 $\mathrm{mm}$ full width at half maximum). Three approaches were used to analyze the fMRI data, and their results were compared.

(1) Statistical analysis of fMRI data was performed with the general linear model (GLM) approach in SPM2 (Friston et al., 1995). For a given patient, the epochs of spike-wave discharges were visually identified and used to construct a boxcar model of active versus rest EEG state, which was then convolved with the canonical hemodynamic response function (HRF) (Glover, 1999) from SPM2. The HRF consists of a standard double gamma function with default parameters documented in the spm_hrf.m file (delay of response relative to onset $=6 \mathrm{~s}$; delay of undershoot relative to onset $=16 \mathrm{~s}$; dispersion of response $=1$; dispersion of undershoot $=1$; ratio of response to undershoot $=6$; onset $=0 \mathrm{~s}$; length of kernel $=32 \mathrm{~s}$ ); this resulted in an HRF peak time of $5.1 \mathrm{~s}$ and an undershoot nadir time of $15.8 \mathrm{~s}$. For fMRI data obtained during CPT or RTT tasks, the $32 \mathrm{~s}$ block design of task alternating with fixation was included in the model to remove effects of the behavioral task from the results. Both the fMRI data and the design matrices were then high-pass filtered at $128 \mathrm{~s}$, and the resulting model was prewhitened by an autocorrelation AR(1) model. No global scaling was used in the present analysis. For group analysis, the one-sample $t$ test using a second-order random effects model was performed to determine regions showing significant fMRI changes across subjects. We applied a false discovery rate (FDR) with corrected $p$ threshold $=0.05$ (Genovese et al., 2002; Langers et al., 2007; Schwartzman et al., 2009) to correct for multiple comparisons, an approach used in some prior fMRI studies of spike-wave discharges (Labate et al., 2005; Moeller et al., 2008b).

All results for functional data are displayed superimposed on the Montreal Neurological Institute brain template "colin27" (single_subj_T1 in SPM) in radiological convention.

(2) We performed a model-free voxel-based analysis of fMRI BOLD signal changes before, during, and after seizures by averaging the time courses of single voxels across patients. The standard $128 \mathrm{~s}$ high-pass filter from SPM (implemented with in-house software) was applied before analysis, without prewhitening. The time course of fMRI percentage change was then calculated for each voxel as $(\mathrm{D} 1-\mathrm{D} 0) / \mathrm{D} 0 \times 100$, where D1 was signal intensity over time and D0 was averaged signal intensity during entire run (406 images). This fMRI time course was analyzed from $-20 \mathrm{~s}$ to $+40 \mathrm{~s}$ relative to each seizure onset. For inclusion in the analysis, we required that the transient movement during the analysis period was $<3 \mathrm{~mm}$ of translation, and $<1^{\circ}$ of rotation. To facilitate temporal alignment across datasets, each time course (acquired with $1.55 \mathrm{~s}$ TR time resolution) was interpolated to obtain values at $0.1 \mathrm{~s}$ time resolution. Like in the EEG analysis, the preictal, ictal, and postictal fMRI time periods were temporally aligned across seizures, and the ictal period of each seizure was scaled to the mean seizure duration (6.6 s). Again, it should be noted that the preictal and postictal time data were not scaled; they were simply aligned in time across seizures by lining up the seizure onset times for preictal data, and by lining up the seizure end times for postictal data. Finally, the mean time course of each voxel was calculated by averaging across seizures for each patient, and then taking the mean across patients. Percentage fMRI change maps were created for the entire brain at $1 \mathrm{~s}$ intervals before, during, and after seizures using the resulting mean time courses.

We expected the contributions of CPT/RTT tasks to the mean fMRI time course to be small since data segments were temporally aligned to seizures, but task block onset times were randomly distributed in the preceding analysis, so would tend to cancel in the mean. To estimate any effects of the CPT/RTT tasks on the mean fMRI time course, we repeated this analysis using identical data segments relative to task blocks, but with no seizures. For each seizure data segment, we identified matching data segments from the same patient with identical duration and identical timing relative to the behavioral task blocks but during a time when no seizure occurred. The analysis described above was repeated to determine the mean fMRI time course in these matching no-seizure data segments.

(3) A mean time course analysis was performed based on specific volumes of interest (VOIs) using MARSBAR (http://marsbar.sourceforge.net/) (TzourioMazoyer et al., 2002). This analysis used the same fMRI data as in the voxelbased analysis described above. First, seven anatomic cortical and subcortical VOIs were selected based on regions showing the largest fMRI changes in the above analysis. These VOIs were constructed from the standard MARSBAR regions as follows (see supplemental Fig. 5, available at www.jneurosci.org as supplemental material): medial and orbital frontal (Cingulum_Ant + Frontal_Sup_Medial + Frontal_Med_Orb + Frontal_Sup_Orb + Rectus); lateral frontal (Frontal_Inf_Oper + Frontal_Inf_Tri + Frontal Mid + Frontal_Sup); lateral temporal (Heschl + Temporal_Sup); parietal [Angular + Parietal_Sup + Parietal_Inf + Supramarginal + Cingulum Post + Cingulum_Mid (posterior half only] + Precuneus); occipital (Calcarine); Rolandic (Paracentral + Precentral + Postcentral, all truncated below SI $52 \mathrm{~mm}$ ); and thalamus (Thalamus). The mean time course of each VOI for a given fMRI run was calculated by averaging the time courses of all voxels in this VOI. The percentage fMRI change was then calculated as $(\mathrm{D} 1-\mathrm{D} 0) / \mathrm{D} 0 \times 100$, where $\mathrm{D} 1$ was the VOI mean signal intensity over time and D0 was averaged signal intensity during the entire run (406 images). As in the voxel-based analysis, we analyzed the time course of each VOI from $-20 \mathrm{~s}$ to $+40 \mathrm{~s}$ relative to seizure onset, temporally aligned data across seizures using the same convention as above, and calculated the mean time course by first averaging across seizures for each patient, and then taking the mean across patients. As described above, we again repeated the same procedure using identical data segments but with no seizures, to estimate any effects of the CPT/RTT tasks on the mean fMRI time course.

The fMRI time courses for the seven anatomical regions were next compared to each other and to the conventional HRF model in terms of overall shape and timing of fMRI increases. The conventional HRF model consisted of the standard double gamma function described above convolved with a boxcar function (ictal period $=1$, all other times $=0$ ) with duration $6.6 \mathrm{~s}$ (mean seizure duration). The amplitude of the canonical HRF obtained from the SPM2 package (in the spm_hrf.m file) was not changed. To evaluate the overall shape and assess the relative contributions of positive and negative components to the fMRI time courses in each region, we calculated a time course polarity index $=$ (peak amplitude - trough amplitude)/(peak amplitude + trough amplitude). Thus, the time course polarity index will be +1.0 for an fMRI time course with entirely positive polarity (i.e., a large positive peak without undershoot or other negative trough components), -1.0 for entirely negative polarity, and 0.0 for a bipolar time course with equal positive peak and negative trough amplitudes. Finally, to statistically compare the early fMRI increases of the seven regions, the positive half-peak times of the individual patient time courses were identified, where for each subject the fMRI signal peak was first defined as the maximum signal during the interval of data being analyzed. Subsequently, a one-way ANOVA followed by Tukey's HSD method for post hoc pairwise comparison 
(SPSS 15.0) was used to compare these positive half-peak times across regions.

We did not regress movement in our fMRI data, but found that movement (as determined by the realignment step in SPM) during the time interval used for data processing was $0-1 \mathrm{~mm}$ for 40 seizures, $1-2 \mathrm{~mm}$ for 6 seizures, and up to $3 \mathrm{~mm}$ for 8 seizures included in the analysis. Movements that occurred did not correlate with the timing of seizures, so would tend to cancel out in the time course averages.

\section{Results}

Spike-wave-discharge bursts of typical absence seizures were observed in EEG data during fMRI scanning in a total of 9 of the 42 patients recruited in the present study. The mean age of these nine patients (three male and six female) at time of study was 11.9 years (range 6-15), and the mean age at the time of first seizure was 8.1 years (range $4-13$ ). In total, 88 seizures were acquired. Seventy-four of the seizures were obtained during CPT or RTT runs, which were performed in all nine patients. Fourteen of the seizures were obtained during VFT runs, which were performed in four of the nine patients.

We attempted to retain as many seizures as possible to maximize the sample size and to keep datasets for the EEG, behavioral, and $\mathrm{fMRI}$ analyses similar. However, we had to eliminate some seizures from behavioral analysis due to lack of behavioral data (no target letter presentations), and from EEG-fMRI analysis due to patient movement $(>3 \mathrm{~mm})$, or insufficient time between seizures to allow for a full time course analysis. For the behavioral analysis, we were able to use 53 of the 88 seizures (eight patients). Forty-one of these seizures were in CPT runs, and twelve were in RTT runs.

For EEG and fMRI analyses, we were able to use 54 of the 88 seizures (nine patients). Forty of these seizures were during CPT or RTT runs (eight patients), and fourteen were in VFT runs (four patients; three patients had seizures during both CPT/RTT and VFT runs). The same 54 seizures were used in the timefrequency analysis of EEG data and in the fMRI analyses, with the exception that an additional three seizures (one patient) were excluded from the fMRI voxel-based and VOI-based time course analyses, since transient movement occurred in this patient during the analysis window for time course analysis, but was well before seizure onset, so could be included in the conventional GLM. On the whole, 54 seizures (nine patients) were used for EEG analysis and GLM-based fMRI analysis, and 51 seizures (eight patients) for voxel-based and VOI-based fMRI time course analyses, resulting in practically the same seizure dataset for the EEG and fMRI-based analyses.

\section{Time course of EEG changes}

The dominant changes in EEG signal power began and ended abruptly with seizures (Fig. 1). EEG recordings in multiple channels showed that all 54 spike-wave discharges were of largest amplitude in the frontal brain regions. Therefore, a Fourier transform was performed to calculate the time-frequency dynamics of the EEG signals in these regions. Figure 1 shows a 2D plot of average time-frequency-power of spike-wave discharges in electrode F7 from -20 to +26 s relative to seizure onset. The results of other channels (F3, F4, FP1, FP2, and F8) are displayed in supplemental Figure 1 (available at www.jneurosci.org as supplemental material). The dominant frequency power increases were observed in the range of 3-4 Hz. The frequency of spikewave discharges at the beginning of the seizure period was maximal at $\sim 4 \mathrm{~Hz}$ and decreased to $\sim 3 \mathrm{~Hz}$ by the end. There were no substantial frequency components seen before or after the seizure period on the timescales relevant for behavioral or fMRI analyses.

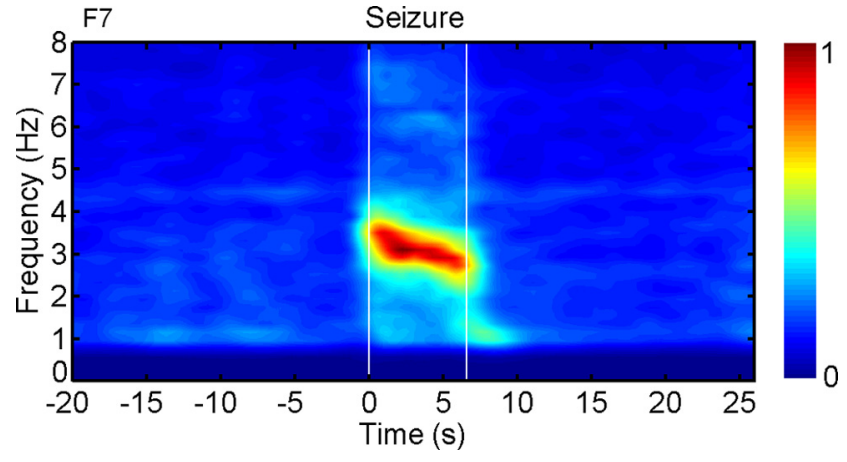

Figure 1. EEG signal power changes abruptly at the beginning and end of seizures. Average time-frequency dynamics of spike-wave discharges in channel F7 channel from -20 s to +26 s relative to seizure onset. A total of 54 seizures (9 patients) were analyzed: 40 seizures in eight patients during CPT or RTT and 14 seizures in four patients during VFT. Analysis was performed by using short-time Fourier transform (see Materials and Methods). All ictal periods were scaled to the mean seizure duration $(6.6 \mathrm{~s})$; and the preictal $(-20$ to $0 \mathrm{~s})$, ictal $(0-6.6 \mathrm{~s})$, and postictal (seizure end to $+26 \mathrm{~s}$ ) periods were then temporally aligned across seizures. The power spectrum was normalized to the range of [0 1] and shown in blue to red scale where the maximum power for each seizure in the range $0-25 \mathrm{~Hz}$ from -20 s to +26 s was defined as " 1 ." Powerfrequency maps for all seizures were averaged across patients. The dominant frequency component of the EEG signal was $3-4 \mathrm{~Hz}$, which was only observed during spike-wave discharges. Average time-frequency dynamics in other frontal channels (FP1, FP2, F3, F4, and F8) are shown in supplemental Figure 1 (available at www.jneurosci.org as supplemental material).

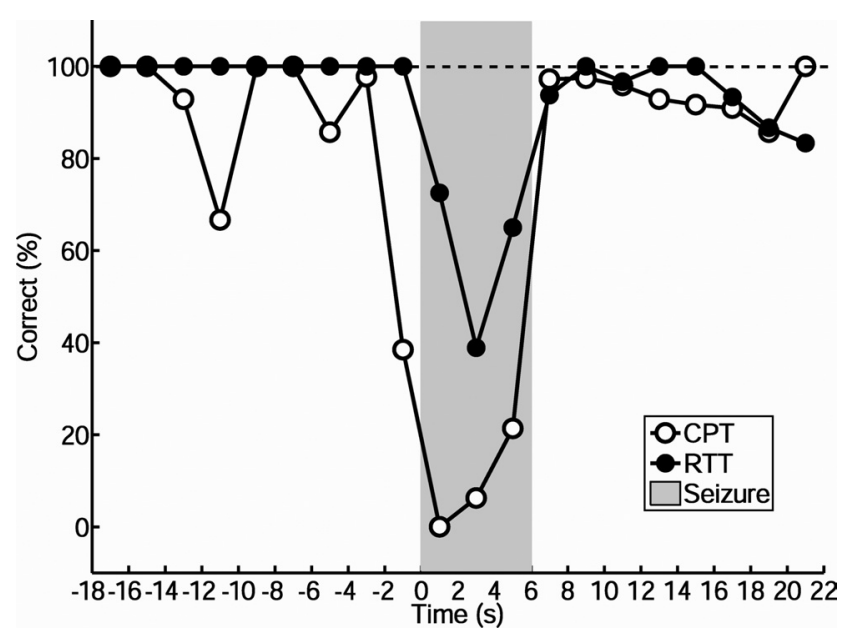

Figure 2. Behavioral impairment during seizures. Percentage correct responses are shown over time ( 2 s time bins) before, during, and after seizures (shaded region). Data were temporally aligned to match the preictal, ictal, and postictal periods across seizures as described in the text. Performance on the more difficult CPT task declined rapidly for letters presented just before seizure onset and recovered quickly after seizures end. Impaired performance on the RTT task was more transient than on CPT, did not begin until after seizure onset, and was less severely impaired during seizures than the (PT task ( $F=15.3, p=0.017$; ANOVA). Results are based on a total of 53 seizures in eight patients: 41 seizures in five patients during CPT and 12 seizures in four patients during RTT. Fluctuations in baseline with CPT appear due to variable and small sample sizes in some (PT bins (e.g., -12 to -10 s bin), since target letters appear on average only once per $4 \mathrm{~s}$ with CPT, while they appear every second with RTT.

\section{Time course of behavioral changes}

Like the EEG changes, behavioral impairment also began and ended relatively abruptly with seizure onset and end (Fig. 2). Behavioral performance was obtained during a total of 53 seizures (eight patients). With CPT testing, percentage correct responses declined rapidly from $98 \%$ at baseline to $38 \%$ for target letters presented even just before seizure onset ( -2 to $0 \mathrm{~s}$ bin) (Fig. 2). During seizures, percentage correct responses on the CPT were markedly depressed at $0 \%, 6 \%$, and $21 \%$ for the three 
time bins during seizures. Responses quickly recovered to $97 \%$ after seizure end (6-8 s bin) (Fig. 2). Impaired performance on the RTT was more transient than on the CPT and did not begin until after seizure onset (0-2 s bin) (Fig. 2); performance again rapidly improved after seizure end. In addition, percentage correct responses on the RTT during seizures $(72 \%, 39 \%, 65 \%)$ were less severely impaired than on the CPT $(F=15.3, p=$ 0.017; ANOVA). Baseline performance before and after seizures was generally $>90 \%$ for both tasks, although some fluctuations in baseline scores appear with CPT due to variable and small sample sizes in some bins (e.g., -12 to $-10 \mathrm{~s}$ bin for CPT). Although the possibility of subtle behavioral impairment before and after seizures cannot be excluded, the most severe deficits in performance clearly occurred during seizures. Scores for the 1 bin just before seizures and the 3 bins during seizures were significantly lower than during the preictal and postictal periods on CPT $(F=73.6, p<0.001$; ANOVA with post hoc pairwise Tukey HSD), and scores on RTT were significantly lower for the three bins during seizures than at other times $(F=16.3, p=0.001)$. Of note, missed CPT responses in the one bin just before seizures do not truly reflect a preictal deficit, since all missed responses in this bin were due to letters that occurred so close to seizures (less than the mean reaction time of $502.9 \mathrm{~ms}$ after the seizure) that the expected response would have had to occur after seizure onset.

\section{fMRI changes based on standard HRF and GLM}

We were interested in determining whether fMRI changes, like EEG and behavioral changes, occur mainly in tight association with seizures or whether they alternatively exhibit a more complicated time course. As a first step, we assumed, as in many other studies (Aghakhani et al., 2004; Laufs et al., 2006; Hamandi et al., 2008; Moeller et al., 2008b), that fMRI changes are governed by a standard hemodynamic response function (Glover, 1999). We further assumed that this HRF can be convolved with a simple boxcar function beginning and ending abruptly with seizure onset and end (representing electrical spike-wave activity) to detect any fMRI changes associated with seizures using a GLM approach. The results of this analysis, combining 54 seizures in nine patients, demonstrate fMRI increases and decreases in regions similar to those reported previously for spikewave seizures (Fig. 3). Thus, we observed fMRI increases in the bilateral thalamus and occipital cortex, with smaller regional increases in the midline cerebellum, anterior and lateral temporal lobes, insula, and the area adjacent to the lateral ventricles. Decreased fMRI signals were observed in the bilateral lateral parietal cortex, precuneus, cingulate gyrus, and anteroventral basal ganglia (Fig. 3). The prominent fMRI increases in bilateral thala- mus and decreases in "default mode" lateral and medial parietal and anterior cingulate cortex are consistent with results reported in other fMRI studies of spike-wave seizures with similar HRF modeling (Aghakhani et al., 2004; Laufs et al., 2006; Hamandi et al., 2008; Moeller et al., 2008a,b).

Time course of fMRI changes using a data-driven approach: voxel-based percentage change maps

The conventional GLM analysis in Figure 3 rests on several untested assumptions. First, it assumes that fMRI changes are directly related to underlying brain electrical activity by the standard Glover HRF model. Second, it assumes that electrical activity in the entire brain starts and ends in a box-car functionlike manner during seizures. To examine fMRI changes without making assumptions, it is necessary to view the data without imposing an a priori model. To accomplish this, we constructed maps of the mean percentage change in fMRI signal on a voxelby-voxel basis throughout the brain before, during, and after 51 seizures in eight patients (Fig. 4). Viewing the data in this manner 


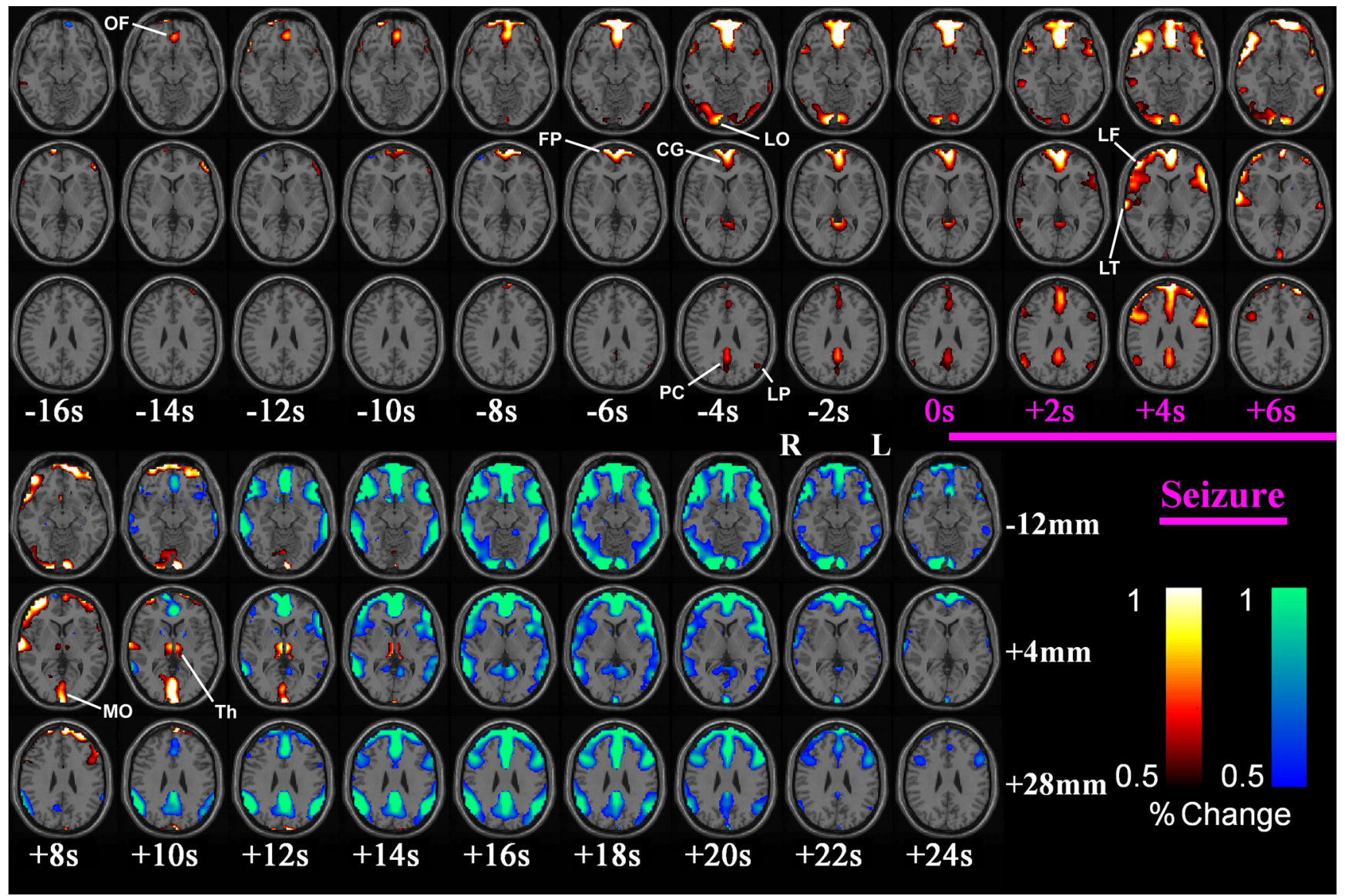

Figure 4. Early and late fMRI changes in cortical-subcortical networks associated with absence seizures Percentage fMRI signal changes are shown from group analysis of 51 seizures in eight patients: 37 seizures in seven patients during (PT or RTT and 14 seizures in four patients during VFT ( 3 patients with seizures during both CPT/RTT and VFT runs). fMRI percentage change increases (warm colors) and decreases (cool colors) are shown, with a display threshold of $0.5 \%$. The ictal time period of seizures was scaled to $6.6 \mathrm{~s}$ (mean seizure duration), and the preictal, ictal, and postictal time periods temporally aligned across all seizures. Early fMRI signal increases were seen well before seizure onset ( 0 s) in medial orbital frontal (OF), frontal polar (FP), cingulate (CG), lateral parietal $(\mathrm{LP})$, precuneus (PC), and lateral occipital (LO) cortex. After seizure onset, fMRI increases progressed to also involve lateral frontal (LF) and temporal (LT) cortex. Following the end of seizures, fMRI increases were seen in the medial occipital (MO) cortex, and lastly in the thalamus (Th). fMRI signal decreases occurred later, and continued well after seizure end. Decreases were seen observed initially in medial/orbital frontal, cingulate, medial, and lateral parietal cortex, followed by decreases in lateral frontal, temporal, and occipital cortex and basal ganglia. See also supplemental Figure 2 and the supplemental video (available at www.jneurosci.org as supplemental material) for more detailed views.

revealed a complex sequence of cortical and subcortical changes both before and after absence seizures not detected by the standard HRF model. Beginning as early as $8-14 \mathrm{~s}$ before seizure onset, positive fMRI changes were observed in cortical regions including the medial orbital frontal, frontal polar, cingulate, lateral parietal, precuneus, and lateral occipital cortex (Fig. 4; see also supplemental Fig. 2, available at www.jneurosci.org as supplemental material). After seizure onset, fMRI increases progressed to also involve lateral frontal and temporal cortex. Following the end of seizures, fMRI increases were seen in the medial occipital cortex and lastly in the thalamus (Fig. 4; see also supplemental Fig. 2, available at www.jneurosci.org as supplemental material).

Negative fMRI responses occurred later, continued long after seizure end, and often followed positive fMRI responses in the same regions. Thus, fMRI decreases were seen initially in medial/ orbital frontal, cingulate, medial, and lateral parietal cortex beginning at approximately $+10 \mathrm{~s}$ after seizure onset, and were followed by decreases in lateral frontal, temporal, and occipital cortex, and basal ganglia (Fig. 4; see also supplemental Fig. 2, available at www.jneurosci.org as supplemental material). The complex pattern of fMRI increases and decreases over time may be more easily appreciated through animated maps (see supple- mental video, available at www.jneurosci.org as supplemental material).

Because most of the fMRI data were obtained during behavioral tasks, it was important to determine whether the observed changes were specifically associated with seizures. Therefore, we repeated the analysis in these patients during times when no seizures occurred by using the same number of data segments and maintaining identical timing with respect to behavioral tasks. In this analysis of nonseizure time intervals, only small transient changes in fMRI signal were observed (see supplemental Figs. 3, 4, available at www.jneurosci.org as supplemental material). Without seizures, we did not observe the sequence of corticalsubcortical fMRI changes seen in identical time segments with seizures (Fig. 4). This suggests that the mean percentage change maps shown in Figure 4 average out signal fluctuations associated with task, leaving the remaining signals specifically associated with spike-wave seizures.

Time course of fMRI changes in identified volumes of interest To determine whether the standard HRF model adequately captures fMRI changes associated with seizures, we calculated mean time courses in representative regions and compared these to the modeled time course. Modeling the fMRI changes with a stan- 
dard Glover HRF convolved by a boxcar function (representing seizure duration) predicts that fMRI signal increases should always begin after EEG seizure onset, have a large positive peak at $9 \mathrm{~s}$, and be followed by a much smaller negative trough $19.8 \mathrm{~s}$ after seizure onset (Fig. 5, red curves representing model). Regions showing fMRI decreases associated with seizures are predicted to simply have an inverted response with a large negative trough at $9 \mathrm{~s}$ followed by a much smaller positive peak $19.8 \mathrm{~s}$ after seizure onset (Fig. 5, blue curves). The large difference between the peak and trough amplitudes in the model yields a predicted time course polarity index of +0.81 for $\mathrm{fMRI}$ increases, and -0.81 for fMRI decreases (Table 1).

We analyzed the fMRI signal time course in seven anatomical regions shown to have large percentage changes associated with seizures (Fig. 4). These anatomical regions were the orbital/medial frontal, lateral frontal, parietal (lateral parietal + precuneus), lateral temporal, occipital, Rolandic cortex, and the thalamus (for regions used, see supplemental Fig. 5, available at www.jneurosci.org as supplemental material). We found that the fMRI time course in most of these regions did not resemble the expected results based on the HRF model (Fig. 5). Although there was considerable variability in signal shape and timing between regions, all regions generally showed an initial fMRI increase, followed by an fMRI decrease, and then recovery back toward baseline. To characterize the timing of the initial fMRI rise, we compared the half-peak times across regions (Table 1). Early fMRI increases (half-peak times) were first seen in the parietal and in the orbital/medial frontal regions at -6.6 and -4.9 s. This was followed by half-peak times of +1.8 $\mathrm{s}$ in the lateral frontal and $+2.4 \mathrm{~s}$ in the lateral temporal regions. Finally, the Rolandic, occipital, and thalamus showed half-peak times of $+3 \mathrm{~s},+4.5 \mathrm{~s}$, and $+6.5 \mathrm{~s}$, respectively. The fMRI decreases (half-trough times) occurred in generally the same sequence as the fMRI increases (Table 1). To identify which regions showed significant increases before others, we performed a oneway ANOVA across patients to compare the positive half-peak times between different regions. Results revealed a significant difference between the seven regions $(F=3.78, p=0.003)$ with post hoc pairwise comparisons showing thalamus to be significantly later than the medial orbital frontal $(p=0.005)$ and parietal $(p=0.021)$ regions. The lateral temporal half-peak times were also significantly later than the medial/orbital frontal cortex $(p=0.036)$.

Interestingly, although the parietal and orbital/medial frontal regions showed early fMRI increases preceding electrical seizure onset, when analyzed with the GLM and canonical HRF, these regions show mainly fMRI decreases (Fig. 3). This is because the initial increases are followed by profound fMRI decreases in these regions, which overlap sufficiently with the negative HRF model (Fig. 5, blue curves) to yield overall fMRI decreases with the conventional model. The relatively large fMRI
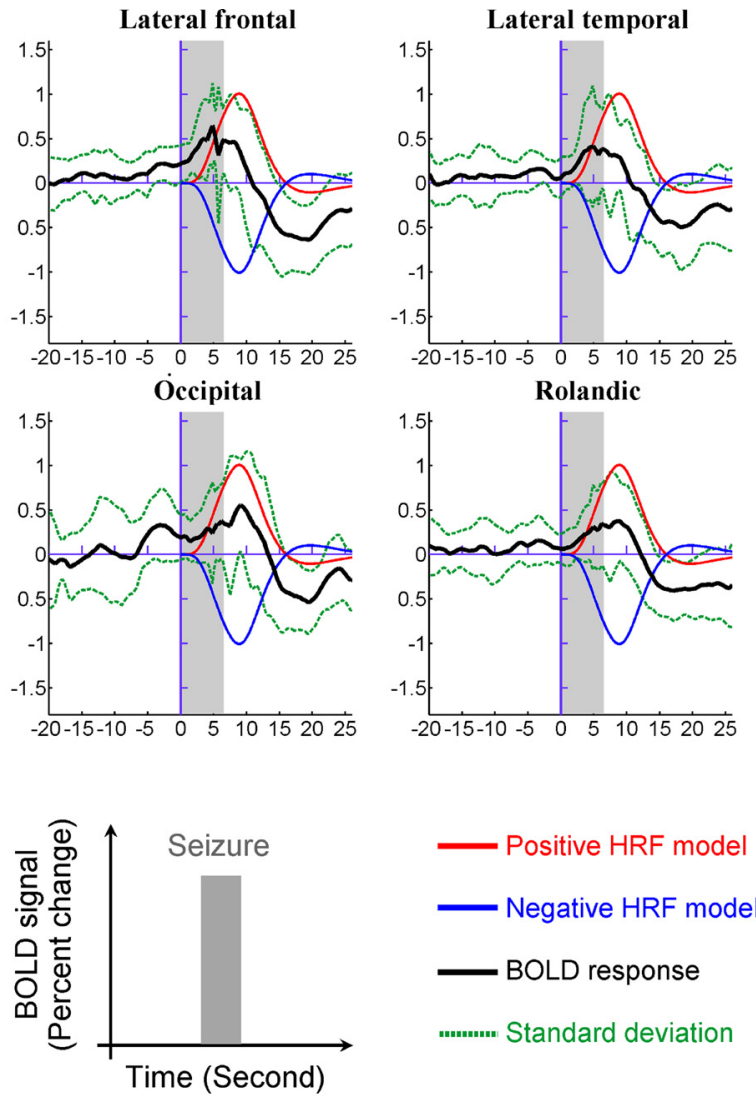

.......... Standard deviation
Figure 5. fMRI time courses during absence seizures differs substantially from model HRF in most regions. Mean fMRI time courses in seven anatomical VOIs (for regions, see supplemental Fig. 5, available at www.jneurosci.org as supplemental material) obtained by convolving a boxcar function of $6.6 \mathrm{~s}$ (mean seizure duration, shown in gray) with the canonical HRF of SPM2. Green
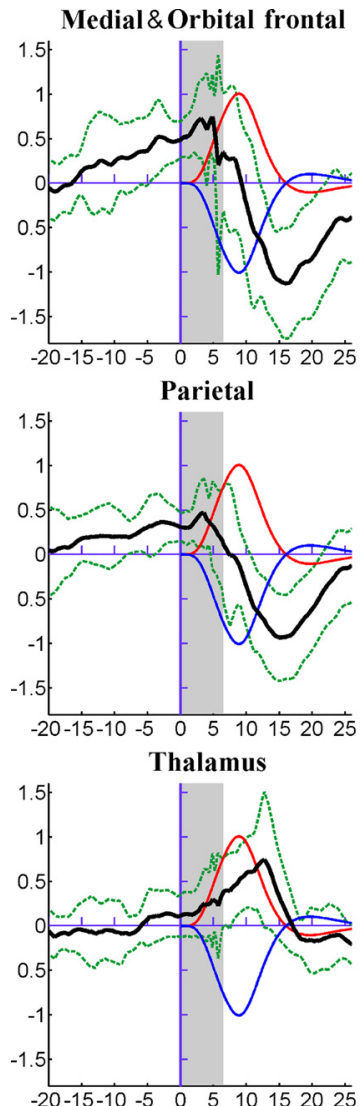

dashed lines are SD. Data are the same as in Figure 4, and were obtained from group analysis of 51 seizures in eight patients.

decreases following the increases in these regions also produce a negative time course polarity index of -0.25 for parietal and -0.20 for orbital/medial frontal cortex, approaching the negative HRF model value of -0.81 more closely than any other regions (Table 1). The lateral frontal and lateral temporal fMRI signal curves have a fairly balanced biphasic shape (time course polarity indices 0.01 and 0.04) (Table 1), and their curves cross the zero level very close to the peak time of the model HRFs (Fig. 5). Therefore, the lateral frontal and temporal cortex are mainly "invisible" with conventional HRF modeling in the GLM (Fig. 3), despite the changes observed in these regions by examining the fMRI time courses directly (Figs. 4, 5). The Rolandic and occipital cortex also showed generally balanced biphasic shapes (time course polarity indices -0.02 and 0.01 ; Table 1 ); however, their curves were shifted later in time (Fig. 5). Therefore, overlap with the positive HRF model (Fig. 5, red curve), particularly for the occipital cortex yielded a large positive signal on conventional analysis (Fig. 3). Of note, the Rolandic cortex signal was below threshold for the GLM model with our data (Fig. 3) but has been reported to be involved in some prior studies using SPM (SalekHaddadi et al., 2003; Aghakhani et al., 2004; Gotman et al., 2005; Laufs et al., 2006; Moeller et al., 2008b), and did show low amplitude increases on percentage change maps in our data (see supplemental Fig. 2, available at www.jneurosci.org as supplemental material). Finally, the thalamus, which is most consistently reported to show fMRI increases during spike-wave seizures, had an fMRI signal shape that was closest to the model HRF (time 
Table 1. Timing and shape of fMRI percentage change time courses for HRF model versus VOI-based data

\begin{tabular}{|c|c|c|c|c|c|}
\hline & 1/2 Peak time & Peak time & $1 / 2$ Trough time & Trough time & Time course polarity index ${ }^{a}$ \\
\hline HRF model, positive ${ }^{b}$ & 5.3 & 9 & 17.1 & 19.8 & 0.81 \\
\hline HRF model, negative ${ }^{b}$ & 17.1 & 19.8 & 5.3 & 9 & -0.81 \\
\hline Parietal & -6.6 & 3.5 & 10.5 & 15.2 & -0.25 \\
\hline Orbital/medial frontal & -4.9 & 4.8 & 11.4 & 15.9 & -0.20 \\
\hline Lateral frontal & 1.8 & 4.8 & 13.4 & 19.3 & 0.01 \\
\hline Lateral temporal & 2.4 & 4.9 & 13.3 & 18.2 & -0.04 \\
\hline Rolandic & 3 & 8.8 & 13.5 & 17.2 & -0.02 \\
\hline Occipital & 4.5 & 9.1 & 14.6 & 19.4 & 0.01 \\
\hline Thalamus & 6.5 & 12.7 & 17.6 & 21 & 0.36 \\
\hline
\end{tabular}

${ }^{a}$ Time course polarity index $=$ (peak amplitude - trough amplitude)/(peak amplitude + trough amplitude). ${ }^{b}$ Positive and negative HRF models are inverted but otherwise identical (see Fig. 5 ).

course polarity index 0.36 ) (Table 1 ; see also Fig. 5) with a relatively late-peaking fMRI increase, leading to a strong positive signal with conventional analysis (Fig. 3 ).

As in the voxel-based percentage change maps, we repeated the analysis using identical data segment timing with respect to task but without seizures. Again, we found only small transient changes in the mean fMRI signal (see supplemental Fig. 6, available at www.jneurosci.org as supplemental material), suggesting that the observed changes in Figure 5 are specifically related to seizures. To determine possible effects of temporal scaling, we also analyzed the same fMRI data before, during, and after seizures without temporal scaling, by simply aligning the seizure onset times (see supplemental Fig. 7, available at www.jneurosci. org as supplemental material). The curves during preictal period were identical to Figure 5 (since no temporal scaling was used in the preictal period). In the ictal and postictal periods there were only small differences, which did not substantially alter the time courses compared to Figure 5. In addition, to evaluate the variability in the fMRI time courses, we calculated the regional time courses during seizures in individual subjects, both during behavioral tasks and during fixation (see supplemental Fig. 8, available at www.jneurosci.org as supplemental material). Although there were considerably more fluctuations in the signal in individual data, and variability between subjects, in most cases the fMRI increases in frontal and parietal cortex occurred relatively early compared to increases in the thalamus.

\section{Discussion}

In the present study, by directly examining the mean fMRI time course in absence seizures, we found evidence for a complex sequence of hemodynamic and metabolic changes that begin and end several seconds beyond electrographic and behavioral alterations. Analysis of EEG showed increases in 3-4 Hz power, and analysis of behavior demonstrated deficits on CPT and RTT tasks, which began and ended abruptly with seizures. In contrast, fMRI increases in the orbital, medial frontal, and parietal cortex began $>5$ $s$ before EEG changes, and widespread fMRI decreases continued for $>20 \mathrm{~s}$ after seizure termination. The time course of fMRI changes in most regions did not, in fact, resemble those expected based on conventional HRF modeling. The thalamus was the one exception, showing a time course similar to the HRF model, and exhibiting the latest peaking $\mathrm{fMRI}$ increases of all regions studied.

These findings suggest that the conventional model, which assumes a boxcar-shaped electrical event convolved with a double gamma function (large positive peak followed by small negative undershoot), does not adequately describe the fMRI changes in absence seizures. This naturally leads to the conclusion that either electrical events in the brain do not begin and end uniformly and abruptly with scalp EEG signals, or the standard HRF does not correctly reflect neurovascular coupling at all places and times, or both.

It is not possible based on the current results to determine whether early fMRI changes - such as those observed in the orbital, medial frontal, and parietal cortex-reflect very early electrical events that cannot be detected on scalp EEG, or whether they arise from primary changes in vascular or metabolic function that precede electrical paroxysms. Of interest, several recent studies have suggested focal cortical abnormalities in human absence seizures, including focal electrical changes in medial or lateral frontal cortex (Holmes et al., 2004; Amor et al., 2009; Westmijse et al., 2009), morphological changes in orbital frontal cortex (Caplan et al., 2009), and possible seizure initiation in medial parietal regions (Vaudano et al., 2009). Similarly, animal models of absence epilepsy have shown focal bilateral cortical abnormalities, which may be crucial for seizure generation (Meeren et al., 2002; Nersesyan et al., 2004; Blumenfeld, 2005a). It important to note, however, that the present results showing cortical fMRI increases many seconds before thalamic increases do not prove that cortical changes "lead" thalamic changes, since the underlying electrical events are not known, and occur over much faster timescales than $\mathrm{PMRI}$ signals. On the contrary, since the thalamus is the one region for which the conventional boxcar-like EEG timing and standard HRF model appear valid, this highlights the importance of the thalamus, at least during the conventional EEG and behavioral time period of absence seizures.

The detailed time course of fMRI changes accompanying absence seizures also sheds light on the results of conventional HRF modeling. Prior studies have found consistent bilateral fMRI increases in the thalamus and decreases in the lateral and medial parietal cortex (Archer et al., 2003; Salek-Haddadi et al., 2003; Aghakhani et al., 2004; Gotman et al., 2005). However, several studies also reported highly variable fMRI increases in bilateral mesial frontal, motor, occipital, and inferior parietal cortex and cerebellum; and decreases in bilateral basal ganglia and frontal and temporal cortex (Salek-Haddadi et al., 2003; Gotman et al., 2005; Laufs et al., 2006; Moeller et al., 2008b). A well known GLM method (Friston et al., 1995) was executed in many of the preceding studies using a canonical HRF (Glover, 1999). Some studies in healthy subjects or animal models (Miezin et al., 2000; Neumann et al., 2003; Handwerker et al., 2004; Meltzer et al., 2008) have noted that the actual hemodynamic response varies from one brain area to another. In recent work on human epilepsy, Gotman and colleagues (Aghakhani et al., 2004; Gotman et al., 2006) applied four single gamma HRFs peaking at 3, 5, 7, and $9 \mathrm{~s}$ after the discharges, along with the canonical HRF. However, variability between different cortical and subcortical brain networks still occurred, suggesting that the actual fMRI BOLD re- 
sponse of some brain regions in spike-wave seizures may differ from the predicted HRFs.

To test for this possibility, we first performed the conventional HRF-based GLM analysis, obtaining fMRI increases mainly in the thalamus, occipital cortex, and cerebellum, and decreases in the bilateral lateral parietal, medial parietal, cingulate, and lateral temporal cortex and basal ganglia, similarly to previous studies (Aghakhani et al., 2004; Gotman et al., 2005; Laufs et al., 2006; Hamandi et al., 2008; Moeller et al., 2008b). On the other hand, percentage fMRI change maps revealed a complex sequence of fMRI increases and decreases, which began before seizure onset and continued for at least $20 \mathrm{~s}$ after seizure end. The fMRI changes of this sequence can be divided into three periods: mainly positive changes from -8 to $+4 \mathrm{~s}$, positive and negative changes from +8 to $+14 \mathrm{~s}$, and only negative changes after +16 $\mathrm{s}$, relative to the seizure onset. Comparing the percentage fMRI change maps over time with the conventional statistical $t$-map, fMRI increases and decreases in the $+12 \mathrm{~s}$ map were very similar to the results obtained by using the GLM method. The fMRI increases in medial/orbital frontal and parietal cortex and decreases in widespread cortical areas were not found in the conventional $t$-map. This confirmed our hypothesis that using predicted HRFs in the analysis led to the omission of some potentially important fMRI changes in the brain. Moreover, the large and long-lasting cortical decrease is also consistent with the results of Gotman and colleagues (Aghakhani et al., 2004; Gotman et al., 2006), who showed the most decreases with an HRF model peaking at 7 or $9 \mathrm{~s}$ after seizures.

Regional time course analyses confirmed early fMRI increases in several cortical areas, and relatively late increases in the thalamus. We observed early fMRI increases in the orbital/medial frontal and medial/lateral parietal cortex $>5 \mathrm{~s}$ before seizure onset. This was followed by profound fMRI decreases in these same regions, which overlapped the timing of the conventional HRF model, likely explaining the "default mode" fMRI decreases typically reported during spike-wave seizures for these regions (Gotman et al., 2005; Laufs et al., 2006). Interestingly, despite the relatively large and widespread fMRI decreases observed for $>20$ $s$ after absence seizure termination, overall brain function is apparently relatively unimpaired, at least with respect to performance on the CPT and RTT tasks, which returns to baseline immediately after electrographic seizures end. The coincidence of the conventional HRF model with the late fMRI decreases (but not earlier increases) in these regions also likely explains the fact that most fMRI studies of spike-wave seizures did not detect the earlier fMRI increases. Other regions showed a more balanced pattern of increases followed by approximately equal decreases. When this pattern coincided with the HRF model peak time, positive and negative changes cancelled, yielding no observable change on conventional modeling (e.g., lateral frontal cortex). When this balanced biphasic pattern occurred later, with the positive phase overlapping with the HRF peak, fMRI increases were seen on conventional modeling (e.g., occipital cortex). Finally, the thalamus showed mainly fMRI increases occurring relatively late in time followed by small decreases, with a time course most similar to the HRF model, yielding large fMRI increases on conventional analysis. These findings reveal a complex and longlasting sequence of fMRI changes in absence seizures, which are not detectable by canonical HRF modeling.

Because early fMRI increases were found in several regions before the onset of spike-wave discharges, we also investigated the time course of EEG and behavioral changes before, during, and after absence seizures. In the EEG analysis, we noted that the predominant time-frequency pattern was of spike-wave discharges in the range of $3-4 \mathrm{~Hz}$ beginning and ending with seizures; changes were not observed in the time range from $20 \mathrm{~s}$ before to $26 \mathrm{~s}$ after seizure onset. Our results are consistent with preceding electrophysiological findings in human absence epilepsy (Bosnyakova et al., 2007; Amor et al., 2009), which have shown early EEG changes on the order of several hundred milliseconds, but not many seconds as seen on fMRI. For behavioral analysis, patient performance revealed impairments that also began and ended abruptly with spike-wave discharges. In addition, absence seizures caused more severe deficits on a CPT than on the simpler RTT task. These results are also compatible with prior behavioral studies showing that most of the severe deficits of absence seizures are confined to the ictal period (Bates, 1953; Tizard and Margerison, 1963; Mirsky and Van Buren, 1965; Goode et al., 1970; Panayiotopoulos et al., 1989). Interestingly, although cessation of performance is clearly associated with the ictal period, some older studies showed more subtle impairments of behavior, which may precede (Mirsky and Rosvold, 1963; Grisell et al., 1964; Mallin et al., 1981) or follow (Lehmann, 1963; Browne et al., 1974; Mallin et al., 1981) absence seizures by a few seconds.

It is important to emphasize that there was considerable variability in the fMRI time courses between subjects, as demonstrated by the relatively large SDs in Figure 5, and the differences between time courses in individual subjects in supplemental Figure 8 (available at www.jneurosci.org as supplemental material). Since childhood absence epilepsy is not a uniformly homogenous disorder, variable patterns of seizure onset may exist between individuals, leading some (for example, see supplemental Fig. $8 b$, available at www.jneurosci.org as supplemental material) to differ substantially from the group mean. Further investigation will be necessary to determine the possible role of this variability in mechanisms of seizure generation, and in variable behavioral deficits during seizures.

In conclusion, our results demonstrate a complex sequence of fMRI changes in absence seizures, which are not detectable by predicted HRF modeling. In addition, we found BOLD fMRI increases in the orbital, medial frontal, and parietal regions before the seizure onset, and large, long-lasting fMRI decreases in widespread brain regions after seizure termination. These results may be important mechanistically for seizure initiation and termination and may also contribute to changes in EEG and behavior.

\section{References}

Aghakhani Y, Bagshaw AP, Bénar CG, Hawco C, Andermann F, Dubeau F, Gotman J (2004) fMRI activation during spike and wave discharges in idiopathic generalized epilepsy. Brain 127:1127-1144.

Amor F, Baillet S, Navarro V, Adam C, Martinerie J, Le Van Quyen M (2009) Cortical local and long-range synchronization interplay in human absence seizure initiation. Neuroimage 45:950-962.

Andermann F (2006) What is a generalized epilepsy? In: Generalized seizures: from clinical phenomenology to underlying systems and networks (Hirsch E, Andermann F, Chauvel P, Engel J, Lopes da Sliva F, Luders H, eds), pp 23-32. Montrouge: John Libbey Eurotex.

Archer JS, Abbott DF, Waites AB, Jackson GD (2003) fMRI "deactivation" of the posterior cingulate during generalized spike and wave. Neuroimage 20:1915-1922.

Barceloux M (1998) Medical toxicology. New York: Elsevier.

Bates JAV (1953) A technique for identifying changes in consciousness. Electroencephalogr Clin Neurophysiol 5:445-446.

Blumenfeld H (2005a) Cellular and network mechanisms of spike-wave seizures. Epilepsia 46 [Suppl 9]:21-33.

Blumenfeld H (2005b) Consciousness and epilepsy: why are patients with absence seizures absent? Prog Brain Res 150:271-286.

Bosnyakova D, Gabova A, Zharikova A, Gnezditski V, Kuznetsova G, van Luijtelaar G (2007) Some peculiarities of time-frequency dynamics of 
spike-wave discharges in humans and rats. Clinical Neurophysiology 118:1736-1743.

Browne TR, Penry JK, Porter RJ, Dreifuss FE (1974) Responsiveness before, during and after spike-wave paroxysms. Neurology 24:659-665.

Buchanan RA, Fernandez L, Kinkel AW (1969) Absorption and elimination of ethosuximide in children. J Clin Pharmacol J New Drugs 9:393-398.

Caplan R, Levitt J, Siddarth P, Wu KN, Gurbani S, Sankar R, Shields WD (2009) Frontal and temporal volumes in childhood absence epilepsy. Epilepsia 50:2466-2472.

Chen C, Casale EJ, Duncan B, Culverhouse EH, Gilman J (1999) Pharmacokinetics of lamotrigine in children in the absence of other antiepileptic drugs. Pharmacotherapy 19:437-441.

Commission on Classification and Terminology of the International League Against Epilepsy (1989) Proposal for revised classification of epilepsies and epileptic syndromes. Epilepsia 30:389-399.

Davidoff RA, Johnson LC (1964) Paroxysmal EEG activity and cognitivemotor performance. Electroencephalogr Clin Neurophysiol 16:343-354.

Friston KJ, Holmes AP, Worsley KP, Poline JB, Frith CD, Frackowiak RSJ (1995) Statistical parametric maps in functional imaging: a general linear approach. Hum Brain Mapp 2:189-210.

Genovese CR, Lazar NA, Nichols T (2002) Thresholding of statistical maps in functional neuroimaging using the false discovery rate. Neuroimage $15: 870-878$.

Glover GH (1999) Deconvolution of impulse response in event-related BOLD fMRI. Neuroimage 9:416-429.

Goode DJ, Penry JK, Dreifuss FE (1970) Effects of paroxysmal spike-wave on continuous visual-motor performance. Epilepsia 11:241-254.

Gotman J, Grova C, Bagshaw A, Kobayashi E, Aghakhani Y, Dubeau F (2005) Generalized epileptic discharges show thalamocortical activation and suspension of the default state of the brain. Proc Natl Acad Sci U S A 102:15236-15240.

Gotman J, Kobayashi E, Bagshaw AP, Bénar CG, Dubeau F (2006) Combining EEG and fMRI: a multimodal tool for epilepsy research. J Magn Reson Imaging 23:906-920.

Grisell JL, Levin SM, Cohen BD, Rodin EA 1964 Effects of subclinical seizure activity on overt behavior. Neurology 14:133-135.

Hamandi K, Salek-Haddadi A, Laufs H, Liston A, Friston K, Fish DR, Duncan JS, Lemieux L (2006) EEG-fMRI of idiopathic and secondarily generalized epilepsies. Neuroimage 31:1700-1710.

Hamandi K, Laufs H, Nöth U, Carmichael DW, Duncan JS, Lemieux L (2008) BOLD and perfusion changes during epileptic generalised spike wave activity. Neuroimage 39:608-618.

Handwerker DA, Ollinger JM, D'Esposito M (2004) Variation of BOLD hemodynamic responses across subjects and brain regions and their effects on statistical analyses. Neuroimage 21:1639-1651.

Hawco CS, Bagshaw AP, Lu Y, Dubeau F, Gotman J (2007) BOLD changes occur prior to epileptic spikes seen on scalp EEG. Neuroimage 35:1450-1458.

Holmes MD, Brown M, Tucker DM (2004) Are "generalized" seizures truly generalized? Evidence of localized mesial frontal and frontopolar discharges in absence. Epilepsia 45:1568-1579.

Hvidberg EF, Dam M (1976) Clinical pharmacokinetics of anticonvulsants. Clin Pharmacokinet 1:161-188.

Jus A, Jus C (1960) Etude electro-clinique des alterations de conscience dans le petit mal. Studii si cercetari de Neurol 5:243-254.

Labate A, Briellmann RS, Abbott DF, Waites AB, Jackson GD (2005) Typical childhood absence seizures are associated with thalamic activation. Epileptic Disord 7:373-377.

Langers DR, Jansen JF, Backes WH (2007) Enhanced signal detection in neuroimaging by means of regional control of the global false discovery rate. Neuroimage 38:43-56.

Laufs H, Lengler U, Hamandi K, Kleinschmidt A, Krakow K (2006) Linking generalized spike-and-wave discharges and resting state brain activity by using EEG/fMRI in a patient with absence seizures. Epilepsia 47:444-448.

Lehmann HJ (1963) Weckreaktionen bei pyknoleptischen absenzen. Arch Psychiat Nervenkr 204:417-426.

Mallin U, Stefan H, Penin H (1981) Psychopathometrische Studien zum Verlauf epileptischer Absencen. Z EEG EMG 12:45-49.

Meeren HK, Pijn JP, Van Luijtelaar EL, Coenen AM, Lopes da Silva FH
(2002) Cortical focus drives widespread corticothalamic networks during spontaneous absence seizures in rats. J Neurosci 22:1480-1495.

Meltzer JA, Negishi M, Constable RT (2008) Biphasic hemodynamic responses influence deactivation and may mask activation in block-design fMRI paradigms. Hum Brain Mapp 29:385-399.

Miezin FM, Maccotta L, Ollinger JM, Petersen SE, Buckner RL (2000) Characterizing the hemodynamic response: effects of presentation rate, sampling procedure, and the possibility of ordering brain activity based on relative timing. Neuroimage 11:735-759.

Mirsky AF, Rosvold HE (1963) Behavioral and physiological studies in impaired attention. In: Psychopharmacological methods: proceedings of a symposium on the effects of psychotropic drugs on higher nervous activity (Votava Z, ed), pp 302-315. Oxford: Pergamon.

Mirsky AF, Van Buren JM (1965) On the nature of the "absence" in centrencephalic epilepsy: a study of some behavioral, electroencephalographic and autonomic factors. Electroencephalogr Clin Neurophysiol 18:334-348.

Moeller F, Siebner HR, Wolff S, Muhle H, Boor R, Granert O, Jansen O, Stephani U, Siniatchkin M (2008a) Changes in activity of striatothalamo-cortical network precede generalized spike wave discharges. Neuroimage 39:1839-1849.

Moeller F, Siebner HR, Wolff S, Muhle H, Granert O, Jansen O, Stephani U, Siniatchkin M (2008b) Simultaneous EEG-fMRI in drug-naive children with newly diagnosed absence epilepsy. Epilepsia 49:1510-1519.

Negishi M, Abildgaard M, Nixon T, Constable RT (2004) Removal of timevarying gradient artifacts from EEG data acquired during continuous fMRI. Clinical Neurophysiology 115:2181-2192.

Negishi M, Abildgaard M, Laufer I, Nixon T, Constable RT (2008) An EEG (electroencephalogram) recording system with carbon wire electrodes for simultaneous EEG-fMRI (functional magnetic resonance imaging) recording. J Neurosci Methods 173:99-107.

Nersesyan H, Hyder F, Rothman DL, Blumenfeld H (2004) Dynamic fMRI and EEG recordings during spike-wave seizures and generalized tonicclonic seizures in WAG/Rij rats. J Cereb Blood Flow Metab 24:589-599.

Neumann J, Lohmann G, Zysset S, von Cramon DY (2003) Within-subject variability of BOLD response dynamics. Neuroimage 19:784-796.

Oppenheim AV, Schafer RW (1989) Discrete-time signal processing. Englewood Cliffs, NJ: Prentice-Hall.

Panayiotopoulos CP, Obeid T, Waheed G (1989) Differentiation of typical absence seizures in epileptic syndromes: a video-EEG study of 124 seizures in 20 patients. Brain 112:1039-1056.

Sadleir LG, Farrell K, Smith S, Connolly MB, Scheffer IE (2006) Electroclinical features of absence seizures in childhood absence epilepsy. Neurology 67:413-418.

Sadleir LG, Scheffer IE, Smith S, Carstensen B, Farrell K, Connolly MB (2009) EEG features of absence seizures in idiopathic generalized epilepsy: impact of syndrome, age, and state. Epilepsia 50:1572-1578.

Salek-Haddadi A, Lemieux L, Merschhemke M, Friston KJ, Duncan JS, Fish DR (2003) Functional magnetic resonance imaging of human absence seizures. Ann Neurol 53:663-667.

Schwartzman A, Dougherty RF, Lee J, Ghahremani D, Taylor JE (2009) Empirical null and false discovery rate analysis in neuroimaging. Neuroimage 44:71-82.

Tizard B, Margerison JH (1963) The relationship between generalized and paroxysmal E.E.G. discharges and various test situations in two epileptic patients. J Neurol Neurosurg Psychiatry 26:308-313.

Tzourio-Mazoyer N, Landeau B, Papathanassiou D, Crivello F, Etard O, Delcroix N, Mazoyer B, Joliot M (2002) Automated anatomical labeling of activations in SPM using a macroscopic anatomical parcellation of the MNI MRI single-subject brain. Neuroimage 15:273-289.

Vaudano AE, Laufs H, Kiebel SJ, Carmichael DW, Hamandi K, Guye M, Thornton R, Rodionov R, Friston KJ, Duncan JS, Lemieux L (2009) Causal hierarchy within the thalamo-cortical network in spike and wave discharges. PLoS One 4:e6475.

Westmijse I, Ossenblok P, Gunning B, van Luijtelaar G (2009) Onset and propagation of spike and slow wave discharges in human absence epilepsy: a MEG study. Epilepsia 50:2538-2548.

Yeager CL, Guerrant JS (1957) Subclinical epileptic seizures; impairment of motor performance and derivative difficulties. Calif Med 86:242-247. 